\title{
Keplerian trigonometry
}

\author{
Alessandro Gambini ${ }^{1}$ - Giorgio Nicoletti ${ }^{2} \cdot$ Daniele Ritelli $^{2}$ (I)
}

Received: 22 June 2020 / Accepted: 5 January 2021 / Published online: 28 January 2021

(c) The Author(s) 2021

\section{Abstract}

Taking the hint from usual parametrization of circle and hyperbola, and inspired by the pathwork initiated by Cayley and Dixon for the parametrization of the "Fermat" elliptic curve $x^{3}+y^{3}=1$, we develop an axiomatic study of what we call "Keplerian maps", that is, functions $\mathbf{m}(\kappa)$ mapping a real interval to a planar curve, whose variable $\kappa$ measures twice the signed area swept out by the $O$-ray when moving from 0 to $\kappa$. Then, given a characterization of k-curves, the images of such maps, we show how to recover the k-map of a given parametric or algebraic k-curve, by means of suitable differential problems.

Keywords Generalized trigonometric functions · Keplerian maps · Eulerian functions · Elliptic integrals · Gauss Hypergeometric function

Mathematics Subject Classification 33B10 $\cdot 33 \mathrm{~B} 15 \cdot 14 \mathrm{H} 52$

\section{Introduction}

In recent years, there has been widespread interest in the possible generalizations of the circular functions. These generalizations are divided into two different lines of thought, depending on the possible fields of application. Some authors, like [7-10,13$17,22]$, taking the moves from the definition of the sine function as inverse of the

Communicated by Adrian Constantin.

$凶$ Daniele Ritelli

daniele.ritelli@unibo.it

Alessandro Gambini

alessandro.gambini@uniroma1.it

Giorgio Nicoletti

giorgio.nicoletti@gmail.com

1 Dipartmento di Matematica Sapienza Università di Roma, Rome, Italy

2 Dipartmento di Scienze Statistiche Università di Bologna, Bologna, Italy 
arcsine function, introduced by the integral

$$
J(u):=\int_{0}^{u}\left(1-t^{2}\right)^{-1 / 2} \mathrm{~d} t
$$

(where $u$ is precisely the arc length of the circle), define the function $\sin _{p}(x)$, where $p \geq 1$, as the inverse of the function

$$
J_{p}(u):=\int_{0}^{u}\left(1-t^{p}\right)^{-1 / p} \mathrm{~d} t,
$$

and then define the function $\cos _{p}$ by placing $\cos _{p}:=\sin _{p}^{\prime}$ : in this manner, they get the identity $\left|\sin _{p}\right|^{p}+\left|\cos _{p}\right|^{p}=1$. This approach is motivated by some analytical properties of these functions, since, adding a second parameter $q$, the functions

$$
J_{p, q}(u):=\int_{0}^{u}\left(1-t^{q}\right)^{-1 / p} \mathrm{~d} t,
$$

are related to the determination of eigenvalues of boundary value problem involving the $p, q$-Laplacian (see [13], eq. (3.9)), i.e. for a problem of the form

$$
\left\{\begin{array}{l}
-\left(\left|u^{\prime}\right|^{p-2}\right)^{\prime}=\lambda|u|^{q-2} u \\
u(a)=u(b)=0 .
\end{array}\right.
$$

With such approach, the parameter $u$ in equation (1) loses its geometric meaning.

On the other side, following the work initiated by Cayley and Dixon $[1,5,6]$ related to Fermat's cubic, $x^{3}+y^{3}=1$, generalized by [11] and more recently in $[3,18,19$, $21,23,24]$ to Fermat curves with arbitrary exponent, other authors define the function pair $\left(\cos _{p}, \sin _{p}\right)$ as the unique solution to the initial value problem

$$
\begin{cases}\phi^{\prime}=-\psi^{p-1}, & \phi(0)=1 \\ \psi^{\prime}=\phi^{p-1}, & \psi(0)=0 .\end{cases}
$$

Such pair of function, as it is easily seen, satisfies the identity $\sin _{p}^{p}+\cos _{p}^{p}=1$.

Although our approach is in line with the latter, we consider the problem from a more general point of view, that goes well beyond Fermat's cubic and Dixon's elliptic functions. In fact, having realized that the variable of the solution $\left(\cos _{p}, \sin _{p}\right)$ to problem (2) measures an area, as for trigonometric and hyperbolic functions, we present a general approach to what we call "Keplerian Trigonometry", that is, the study of a wide class of planar curves admitting a (unique!) parametric representation $\mathbf{m}_{\mathscr{C}}(\kappa)=\cos \mathscr{C}(\kappa) \mathbf{i}+\sin _{\mathscr{C}}(\kappa) \mathbf{j}$, whose components share various properties of usual trigonometric functions, and the parameter $\kappa$ measures twice the signed area swept out by the $O P$-ray when the point $P$ moves along the curve from the unit point of the $X$-axis. 
Our work also includes a section dedicated to a combinatoric version of the problem with a combinatorics of the nonlinear differential system that takes up the particular cases of Fermat curves treated in [24].

In conclusion of the article, we apply the theoretical results to a pair of third-degree (elliptic) plane curves, linked in a similar way to that which connects trigonometric and hyperbolic functions.

\section{Keplerian maps}

An analytic vector function $\mathbf{f}(s):=f_{x}(s) \mathbf{i}+f_{y}(s) \mathbf{j}^{1}$, defined on a real interval $I$, will be called a planar map, or simply a map, and its image $\mathscr{C}:=\mathbf{f}(I)$ will be called a parametric curve, or $p$-curve. Obviously, a second map g, defined on the real interval $J$, parametrizes the same curve $\mathscr{C}$ if, and only if, if there exists a differentiable bijective function $s(t): J \rightarrow I$ such that $s^{\prime} \neq 0$ and $\mathbf{g}(t)=\mathbf{f}(s(t))$. Note that, by our definition, every p-curve consists of only one branch.

In order to focus a wide class of maps that behave like the trigonometric map

$$
\mathbf{t}(\kappa):=\cos (\kappa) \mathbf{i}+\sin (\kappa) \mathbf{j},
$$

(The trigonometric map)

and the hyperbolic map

$$
\mathbf{h}(\kappa):=\cosh (\kappa) \mathbf{i}+\sinh (\kappa) \mathbf{j},
$$

(The hyperbolic map)

we observe that, in addition to mapping 0 to $\mathbf{i}$, for both of them, the variable is Keplerian, that is, it renders twice the signed area swept out by the $O P$-ray when moving from the unit point $U_{x}$ of the $X$-axis to the image of $\kappa$. Hence, it seems natural to consider the class of maps sharing that properties.

To provide a more precise wording of our aim, it is helpful to define the wedge operation $\wedge: \mathbb{R}^{2} \times \mathbb{R}^{2} \rightarrow \mathbb{R}$ by setting, for every $\mathbf{u}, \mathbf{v} \in \mathbb{R}^{2}$ :

$$
\mathbf{u} \wedge \mathbf{v}:=\operatorname{det}\left[\begin{array}{ll}
u_{x} & v_{x} \\
u_{y} & v_{y}
\end{array}\right] .
$$

The scalar $\mathbf{u} \wedge \mathbf{v}$ measures the signed area of the oriented parallelogram with sides $\mathbf{u}, \mathbf{v}$. The fact of being Keplerian the variable $\kappa$ of a map $\mathbf{f}$, is expressed by the identity

$$
\mathrm{d} \kappa=\mathbf{f}(\kappa) \wedge \mathbf{f}(\kappa+\mathrm{d} \kappa)
$$

or better, by its equivalent ${ }^{2}$

$$
\mathbf{f} \wedge \mathbf{f}^{\prime}=1 \text {. }
$$

Here then, the maps of this new class are defined as follows.

\footnotetext{
${ }^{1}$ In this paper, the elements of $\mathbb{R}^{2}$ are presented as column vectors, and the standard basis is denoted by $(\mathbf{i}, \mathbf{j})$.

${ }^{2}$ When, as in this case, the variable is left out, the identity holds for every element of the domain of the function.
} 
Definition 2.1 A map $\mathbf{m}: I \rightarrow \mathbb{R}^{2}$, where $0 \in I$, satisfying the following "Keplerian analytic axioms"

$$
\left\{\begin{array}{l}
\mathbf{m}(0)=\mathbf{i} \\
\mathbf{m} \wedge \mathbf{m}^{\prime}=1
\end{array}\right.
$$

is called a Keplerian map (k-map for short). A k-map $\mathbf{m}$ is called upright if the derivative of its first component vanishes at 0 , that is, if $m_{x}^{\prime}(0)=0$.

The image of a k-map $\mathbf{m}$ is called a Keplerian curve or $k$-curve.

In order to emphasise the analogy with trigonometric functions, the components of the k-map of a given k-curve $\mathscr{C}$ are denoted as $\cos \mathscr{C}$ and $\sin _{\mathscr{C}}$, that is, we will set:

$$
\mathbf{m}_{\mathscr{C}}(\kappa)=\cos \mathscr{C}(\kappa) \mathbf{i}+\sin _{\mathscr{C}}(\kappa) \mathbf{j} .
$$

We will refer to the component $\sin _{\mathscr{C}}$ as to a $\sin$-partner of $\cos \mathscr{C}$, and symmetrically for the component $\cos \mathscr{C}$.

Thanks to the identity $\left(\mathbf{f} \wedge \mathbf{f}^{\prime}\right)^{\prime}=\mathbf{f}^{\prime} \wedge \mathbf{f}^{\prime}+\mathbf{f} \wedge \mathbf{f}^{\prime \prime}=\mathbf{f} \wedge \mathbf{f}^{\prime \prime}$, the Keplerian axioms can be restated in terms of the second derivative.

Theorem 2.2 A map $\mathbf{m}: I \rightarrow \mathbb{R}^{2}$, where $0 \in I$, is a Keplerian map, whenever it satisfies the following axioms:

$$
\left\{\begin{array}{l}
\mathbf{m}(0)=\mathbf{i} \\
\mathbf{m} \wedge \mathbf{m}^{\prime \prime}=0 \\
\mathbf{m}(0) \wedge \mathbf{m}^{\prime}(0)=1
\end{array}\right.
$$

Axiom (AnK 2) states that vectors $\mathbf{m}(\kappa)$ and $\mathbf{m}^{\prime \prime}(\kappa)$ are parallel, so there exists an analytic function $\chi(\kappa)$ such that $\mathbf{m}^{\prime \prime}(\kappa)=\chi(\kappa) \mathbf{m}(\kappa)$, where

$$
\chi=\chi \mathbf{m} \wedge \mathbf{m}^{\prime}=\mathbf{m}^{\prime \prime} \wedge \mathbf{m}^{\prime}
$$

Actually, the preceding theorem has the following result as converse.

Theorem 2.3 Let $\chi: I \rightarrow \mathbb{R}$ be an analytic function such that $0 \in I$; then there exists exactly one Keplerian map $\mathbf{m}$ such that $\mathbf{m}^{\prime \prime}(\kappa)=\chi(\kappa) \mathbf{m}(\kappa)$ for every $\kappa \in I$.

Proof The k-map $\mathbf{m}$ is the solution of the problem

$$
\left\{\begin{array}{l}
\mathbf{p}^{\prime \prime}=\chi \mathbf{p} \\
\mathbf{p}(0)=\mathbf{i} \\
\mathbf{p}(0) \wedge \mathbf{p}^{\prime}(0)=1
\end{array}\right.
$$


The preceding result is the Keplerian analog of the fundamental theorem of the local theory of curves; here the function $\chi$ plays the role of the curvature: then, it seems to be consistent to call $\chi$ the Keplerian curvature of the curve $\mathscr{C}$.

The following result, whose proof is quite elementary, characterises the k-curves, by showing how the (unique) k-map associated to a suitable p-curve can be computed by inverting an integral.

Theorem 2.4 A parametric curve $\mathscr{C}=\mathbf{f}(I)$ is a Keplerian curve if, and only if, there exists $s_{0} \in I$ such that

$$
\mathbf{f}\left(s_{0}\right)=\mathbf{i}
$$

and, for every $s \in I$ :

$$
\mathbf{f}(s) \wedge \mathbf{f}^{\prime}(s) \neq 0 .
$$

Moreover, the Keplerian map which parametrize $\mathscr{C}$ is provided by the map

$$
\mathbf{m}_{\mathscr{C}}(\kappa):=\mathbf{f}(s(\kappa))
$$

where $s(\kappa)$ is the inverse of the function

$$
\kappa(s):=\int_{s_{0}}^{s} \mathbf{f}(u) \wedge \mathbf{f}^{\prime}(u) \mathrm{d} u .
$$

The preceding result can be restated in terms of elementary geometry, by saying that a k-curve has a unique branch, it includes the unit point $U_{x}$, and every its tangent line avoids the origin (Figs. 1, 2, 3 and 4).

As an application of the previous result, let us consider the unit circle involute $\mathscr{C}$, image of the map

$$
\mathbf{f}(t):=(\cos t+t \sin t) \mathbf{i}+(\sin t-t \cos t) \mathbf{j}, \quad t \geq 0 .
$$

We get $\mathbf{f} \wedge \mathbf{f}^{\prime}=t^{2}$, then $\kappa=t^{3} / 3, \quad t=(3 \kappa)^{1 / 3}$, and $\mathbf{m}_{\mathscr{C}}(\kappa)=\mathbf{f}(3 \kappa)^{1 / 3}$.

\section{General identities}

For any given k-curve $\mathscr{C}$, we will set $\tan _{\mathscr{C}}:=\sin _{\mathscr{C}} / \cos _{\mathscr{C}}$; however, we need to remark that the geometric meaning of the trigonometric tangent function is preserved only in case of upright k-curves.

As easy consequences of Keplerian axioms, we have the identities

$$
\begin{array}{ll}
\sin _{\mathscr{C}}^{\prime}(0)=1, & \sin _{\mathscr{C}}^{\prime \prime}(0)=\sin _{\mathscr{C}}(0)=0, \\
\tan _{\mathscr{C}}^{\prime}(0)=1, & \tan _{\mathscr{C}}^{\prime \prime}(0)=\tan _{\mathscr{C}}(0)=0 .
\end{array}
$$

Moreover, it is worthwhile to note that a k-curve $\mathscr{C}$ is a upright one if, and only if, $\cos _{\mathscr{C}}^{\prime}(0)=0$. Again, from (AnK 2) we get immediately the following identities, which 


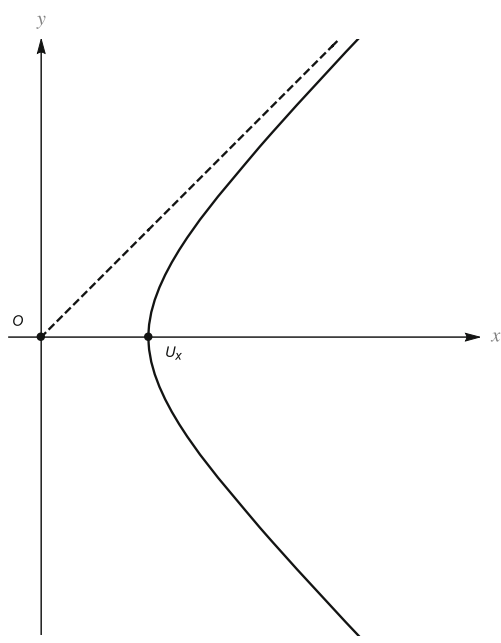

Fig. 1 A keplerian curve

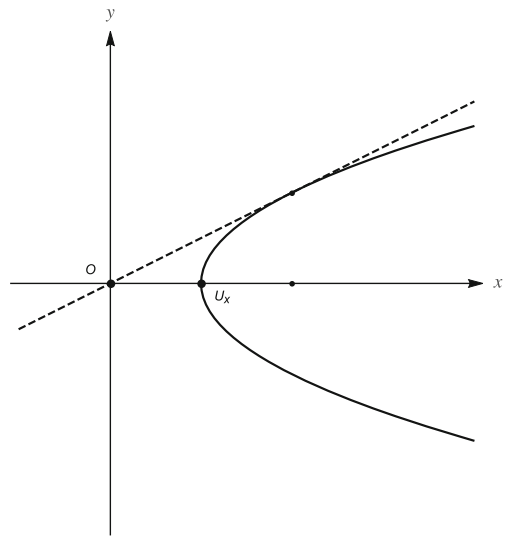

Fig. 2 A non keplerian curve

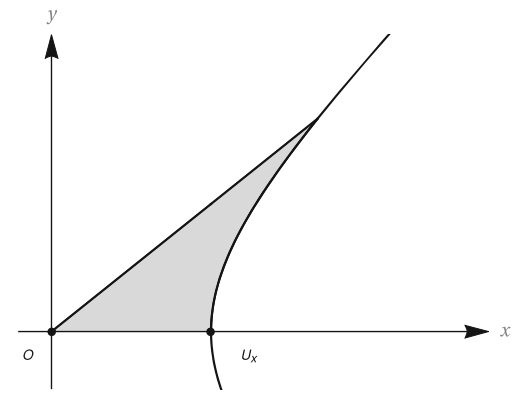

Fig. 3 The Keplerian parameter 


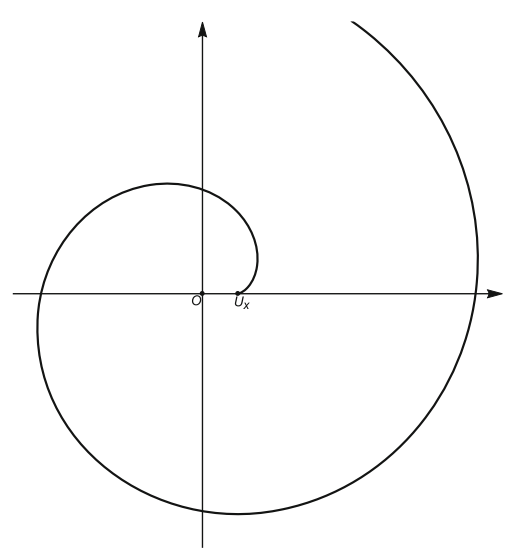

Fig. 4 The unit circle involute

generalize well-known relations of circular and hyperbolic functions:

$$
\begin{aligned}
\tan _{\mathscr{C}}(\kappa) & =\frac{\sin _{\mathscr{C}}(\kappa)}{\cos \mathscr{C}(\kappa)}=\frac{\sin _{\mathscr{C}}^{\prime \prime}(\kappa)}{\cos _{\mathscr{C}}^{\prime \prime}(\kappa)}, \\
\tan _{\mathscr{C}}^{\prime}(\kappa) & =\frac{1}{\cos _{\mathscr{C}}^{2}(\kappa)}, \\
\tan _{\mathscr{C}}(\kappa) & =\int_{0}^{\kappa} \frac{1}{\cos _{\mathscr{C}}^{2}(u)} \mathrm{d} u, \\
\sin _{\mathscr{C}}(\kappa) & =\cos \mathscr{C}(\kappa) \int_{0}^{\kappa} \frac{1}{\cos _{\mathscr{C}}^{2}(u)} \mathrm{d} u .
\end{aligned}
$$

By the last identity, every analytic function $x(t)$, with $x(0)=1$, has exactly one sinpartner; on the contrary, any analytic function $y(t)$, with $y(0)=0, y^{\prime}(0)=1, y^{\prime \prime}(0)=$ 0 , admitting a cos-partner $x(t)$, has all functions $x(t)+\mu y(t)$ as additional cos-partner. Obviously, "monogamy" is restored when the search of partnership is restricted to upright cos-partners.

By axiom (AnK 1), any point $\bar{P}:=\mathbf{m}_{\mathscr{C}}(\bar{\kappa})$ of the curve $\mathscr{C}$ belongs to the line $\sin _{\mathscr{C}}^{\prime}(\bar{\kappa}) x-\cos _{\mathscr{C}}^{\prime}(\bar{\kappa}) y=1$, which turns to be the tangent line to $\mathscr{C}$ at $\bar{P}$, intersecting the $X$-axis at $1 / \sin _{\mathscr{C}}^{\prime}(\bar{\kappa})$ and the $Y$-axis at $-1 / \cos _{\mathscr{C}}^{\prime}(\bar{\kappa})$. By this fact, we are led to define the secant and cosecant functions for the k-curve $\mathscr{C}$ by setting

$$
\begin{aligned}
\sec _{\mathscr{C}}(\kappa) & :=\frac{1}{\sin _{\mathscr{C}}^{\prime}(\kappa)}, \\
\csc _{\mathscr{C}}(\kappa) & :=-\frac{1}{\cos _{\mathscr{C}}^{\prime}(\kappa)} .
\end{aligned}
$$

Remark that for the circle and the hyperbola such definitions return the usual secant and cosecant functions. 
Finally, we remark that if $\mathscr{C}$ is a closed k-curve, its k-map is a periodic function, whose fundamental period $\Pi_{\mathscr{C}}$ is twice the area $\pi_{\mathscr{C}}$ of the region bounded by $\mathscr{C}$.

\section{The arithmetic axioms-formal Keplerian maps}

The analytic Keplerian axiom has a useful arithmetic counterpart.

Theorem 4.1 (The Arithmetic Keplerian Axioms) An analytic vector function $\mathbf{f}(t):=\sum_{i} x_{i} \frac{t^{i}}{i !} \mathbf{i}+\sum_{i} y_{i} \frac{t^{i}}{i !} \mathbf{j}$ is a Keplerian map if, and only if, the following identities hold:

$$
\left\{\begin{array}{c}
\sum_{\substack{i+j=h \\
h \geq 2}}(j-i)\left(\begin{array}{l}
h \\
i
\end{array}\right) x_{i} y_{j}=0 \\
x_{0}=y_{1}=1 \\
y_{0}=y_{2}=0
\end{array}\right.
$$

Proof Let $\mathbf{f}(t) \wedge \mathbf{f}^{\prime}(t)=\sum_{i} w_{i} \frac{t^{i}}{i !}$; then, for every $h \in \mathbb{N}$, we have:

$$
\begin{aligned}
& w_{h}=\left.\mathrm{D}^{h}\left(x(t) y^{\prime}(t)-x^{\prime}(t) y(t)\right)\right|_{t=0}=\sum_{i+j=h}\left(\begin{array}{l}
h \\
i
\end{array}\right)\left(\begin{array}{ll}
x_{i} & \left.y_{j+1}-x_{i+1} y_{j}\right)
\end{array}\right. \\
& =\sum_{i+j=h} \frac{h+1-i}{h+1}\left(\begin{array}{c}
h+1 \\
i
\end{array}\right) x_{i} y_{j+1}-\sum_{i+j=h} \frac{h+1-j}{h+1}\left(\begin{array}{c}
h+1 \\
i
\end{array}\right) x_{i+1} y_{j} \\
& =\frac{1}{h+1} \sum_{i+j=h+1}(j-i)\left(\begin{array}{c}
h+1 \\
i
\end{array}\right) x_{i} y_{j}
\end{aligned}
$$

and the statement follows immediately.

This new dress of the Keplerian Axioms evokes the opportunity to neglect convergence problems, by enlarging our concern to pairs of formal (exponential) series; to do that, we define a formal Keplerian map as an ordered pair

$$
\mathbf{f}(t):=\left(\sum_{i} x_{i} \frac{t^{i}}{i !}, \sum_{i} y_{i} \frac{t^{i}}{i !}\right)
$$

satisfying the Arithmetic Keplerian Axioms.

The following statement is an immediate outcome of Theorem 4.1.

Corollary 4.2 Let $\mathbf{f}(t):=\left(\sum_{i} x_{i} \frac{t^{i}}{i !}, \sum_{i} y_{i} \frac{t^{i}}{i !}\right)$ be a formal $k$-map; then, the following identities hold:

$$
y_{h}=\frac{1}{h} \sum_{\substack{i+j=h \\
0 \neq j \neq h}}(i-j)\left(\begin{array}{l}
h \\
i
\end{array}\right) x_{i} y_{j}, \quad \text { for every } h \geq 2,
$$


and, for every $h \geq 3$ :

$$
x_{h-1}=\frac{1}{h(h-2)} \sum_{\substack{i+j=h \\
0 \neq j \neq 1}}(j-i)\left(\begin{array}{l}
h \\
i
\end{array}\right) x_{i} y_{j}, \quad \text { for every } h \geq 3 .
$$

Identity (4) allow us to compute the unique sin-partner $y(t)$ of any formal series $x(t)$ with $x_{0}=1$; note that in any case will be $y_{2}=0$. Similarly, identity (5) can be employed to compute a cos-partner $x(t)$ of any sequence $y(t)$, with $y_{0}=0, y_{1}=$ $1, y_{2}=0$, only after the choice of $x_{1} \in \mathbb{R}$.

As an application, let us compute the sin-partner $y(t)$ of the function $x(t):=1+a \frac{t^{n}}{n !}$; Theorem 4.1 forces the identities

$$
\begin{aligned}
& y_{1}=1, \\
& y_{n+1}=(n-1) a, \\
& y_{i}=0 \quad \text { whenever } i \neq 1(\bmod n), \\
& y_{(j+1) n+1}=-a \frac{((j-1) n+1)}{n !} \frac{((j+1) n) !}{(j n+1) !} y_{j n+1} .
\end{aligned}
$$

The series $y(t)$ can be now redrafted as follows:

$$
\begin{aligned}
y(t) & =\sum_{j \geq 0} y_{j n+1} \frac{t^{j n+1}}{(j n+1) !} \\
& =\sum_{j \geq 0} \frac{(-n+1)}{((j-1) n+1)} \frac{(j n) !}{(n !)^{j}}(-a)^{j} \frac{t^{j n+1}}{(j n+1) !} \\
& =t \sum_{j \geq 0} \frac{(-n+1)}{((j-1) n+1)} \frac{j !}{(j n+1)} \frac{\left(-a t^{n} / n !\right)^{j}}{j !} .
\end{aligned}
$$

As the ratio $r(j)$ of two consecutive coefficients in the last series is the rational function $r(j)=\left(j+\frac{1}{n}-1\right)(j+1) /\left(j+\frac{1}{n}+1\right)$, we infer that such series is the Gauss hypergeometric function

$$
y(t)=t_{2} \mathrm{~F}_{1}\left(\begin{array}{c}
\frac{1}{n}-1,1 \\
\frac{1}{n}+1
\end{array} \mid-a \frac{t^{n}}{n !}\right) .
$$

\section{Finding the k-map of an algebraic curve}

Our purpose is now to extend the preceding ideas to algebraic curves. Obviously, in this case, too, we must narrow our attention to Keplerian algebraic curves, that is, to algebraic curves containing the point $U_{x}$, whose tangent lines avoid the origin; this 
second condition is equivalent to claim that for every point $P:=(x, y)$ in $\mathscr{C}$ vectors $O P$ and $\nabla f$ are not perpendicular, that is: $x f_{x}+y f_{y} \neq 0$.

The following theorem characterises k-algebraic curves and shows that the unique $\mathrm{k}$-map of a given k-algebraic curve is the solution of a first-order differential problem.

Theorem 5.1 Let the function $f: \mathbb{R}^{2} \rightarrow \mathbb{R}$ satisfy conditions $f(1,0)=0$ and $x f_{x}+$ $y f_{y} \neq 0$; then the algebraic curve $\mathscr{C}:=\left\{(x, y) \in \mathbb{R}^{2} \mid f(x, y)=0\right\}$ is Keplerian, and its $k$-map is the solution $\mathbf{m}_{\mathscr{C}}$ of the differential system

$$
\begin{cases}x^{\prime}=-\frac{f_{y}}{x f_{x}+y f_{y}}, & x(0)=1, \\ y^{\prime}=\frac{f_{x}}{x f_{x}+y f_{y}}, & y(0)=0 .\end{cases}
$$

Proof Conditions on $f$ ensure that $\mathscr{C}$ is a Keplerian curve.

The solution $\mathbf{p}(t)=x(t) \mathbf{i}+y(t) \mathbf{j}$ of system (6) is a k-map, as $\mathbf{p}(0)=\mathbf{i}$, and $\mathbf{p} \wedge \mathbf{p}^{\prime}=1$.

Moreover, we have $f(x(0), y(0))=0$ and $\frac{\mathrm{d} f}{\mathrm{~d} t}=f_{x} \frac{\mathrm{d} x}{\mathrm{~d} t}+f_{y} \frac{\mathrm{d} y}{\mathrm{~d} t}=0$, hence, for every $t$ in a suitable neighbourhood of 0 , it is $f(x(t), y(t))=0$, and $\mathbf{p}=\mathbf{m}_{\mathscr{C}}$.

Differentiating the system (6), and after some elementary calculus, we obtain a second rule for computing the k-map of a k-algebraic curve.

Theorem 5.2 The k-map of a Keplerian algebraic curve $\mathscr{C}:=\left\{(x, y) \in \mathbb{R}^{2} \mid\right.$ $f(x, y)=0\}$ is the solution of the second-order problem

$$
\begin{cases}x^{\prime \prime}=-f_{\triangle} x, & x(0)=1 \\ y^{\prime \prime}=-f_{\triangle} y, & y(0)=0, \quad y^{\prime}(0)=1,\end{cases}
$$

where

$$
\begin{aligned}
f_{\triangle} & :=\frac{f_{x x} f_{y}^{2}-2 f_{x y} f_{x} f_{y}+f_{y y} f_{x}^{2}}{\left(x f_{x}+y f_{y}\right)^{3}} \\
& =\frac{\left[-f_{y} f_{x}\right]\left[\begin{array}{c}
f_{x x} f_{x y} \\
f_{x y} f_{y y}
\end{array}\right]\left[\begin{array}{c}
-f_{y} \\
f_{x}
\end{array}\right]}{\left(x f_{x}+y f_{y}\right)^{3}} .
\end{aligned}
$$

Note that the function $-f_{\triangle}$ is precisely the Keplerian curvature of $\mathscr{C}$ and the numerator is called offline hessian.

\section{1 p-algebraic curves}

When the curve $\mathscr{C}$ has equation $f(x, y)=1$, where $f$ is the irreducible homogeneous polynomial

$$
f(x, y):=\sum_{i=0}^{p} f_{i} x^{p-i} y^{i} \quad f_{0}=1,
$$


we have $x f_{x}+y f_{y}=p$, and the system (6) becomes

$$
\begin{cases}x^{\prime}=-\frac{1}{p} f_{y} & x(0)=1, \\ y^{\prime}=\frac{1}{p} f_{x} & y(0)=0\end{cases}
$$

showing that the derivatives of $x=\cos \mathscr{C}$ and $y=\sin _{\mathscr{C}}$ are the homogeneous polynomials

$$
\begin{aligned}
& x^{\prime}=-\frac{1}{p} \sum_{i=1}^{p} i f_{i} x^{p-i} y^{i-1}, \\
& y^{\prime}=\frac{1}{p} \sum_{i=0}^{p-1}(p-i) f_{i} x^{p-i-1} y^{i},
\end{aligned}
$$

of degree $p-1^{3}$. By repeatedly differentiating, we obtain the following theorem.

Theorem 5.3 Let the $\mathscr{C}:=\{f(x, y)=1\}$ where $f$ is the irreducible homogeneous polynomial

$$
f(x, y):=\sum_{i=0}^{p} f_{i} x^{p-i} y^{i} \quad f_{0}=1 ;
$$

then, the nth derivatives of $x=\cos \mathscr{C}$ and $y=\sin _{\mathscr{C}}$ are homogeneous polynomials of degree $n(p-2)+1$ in variables $x, y$. Furthermore, for every natural $m, n$, we will have

$$
\left(x^{m} y^{n}\right)^{\prime}=\frac{1}{p} \sum_{i=0}^{p}(n(p-i)-m i) f_{i} x^{m+p-i-1} y^{n+i-1} .
$$

Let us now define the the $\mathbb{N} \times \mathbb{N}$ matrix $\mathfrak{M}_{\cos \mathscr{C}}$ by setting $\mathfrak{M}_{\cos \mathscr{C}}(0, i):=\delta_{0, i}$ and by neatly arranging in the $n t h$ row the coefficients of the $n t h$ derivative of $\cos \mathscr{C}$; then, the coefficients of Maclaurin series of $\cos \mathscr{C}$ are the entries in the 0 th column of $\mathfrak{M}_{\cos \mathscr{C}}$, which can be recursively computed by the inverse of the law in identity (8). Analogous arguments hold for the matrix $\mathfrak{M}_{\sin _{\mathscr{C}}}$, defined by setting $\mathfrak{M}_{\sin _{\mathscr{C}}}(0, i):=\delta_{1, i}$.

\section{Some applications}

\subsection{A "dumpy" cubic}

As an instance of application of preceding equations, let us consider the smooth cubic $\mathscr{D}$ of cartesian equation

$$
x^{3}+3 x y^{2}-1=0 .
$$

\footnotetext{
3 For the Fermat curve $\mathscr{F}_{p}$, system (7) becomes $x^{\prime}=-y^{p-1}, y^{\prime}=x^{p-1}$, whose solution, when $p=3$, is the pair of the so-called Dixonian functions $\mathrm{cm}, \mathrm{sm}[1,5,6,11,19]$.
} 


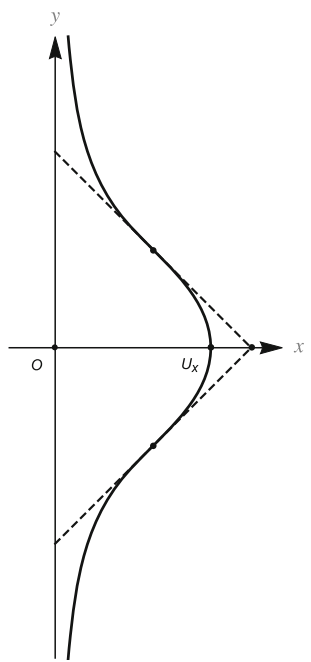

Fig. 5 The curve $x^{3}+3 x y^{2}=1$

Table 1 First coefficients of $\cos \mathscr{D}$ and $\sin \mathscr{D}$

$c_{0}=1 c_{2}=-2 c_{4}=16 c_{6}=-320 c_{8}=12160 c_{10}=-742400 c_{12}=66457600 c_{14}=-8202444800$

$s_{1}=1 s_{3}=-2 s_{5}=40 s_{7}=-1040 s_{9}=52480 s_{11}=-3872000 s_{13}=411136000 s_{15}=-58479872000$

Such k-curve is projectively closed and symmetric with respect to the $\mathrm{X}$-axis ${ }^{4}$ : consequently, $\cos \mathscr{D} \kappa=: \sum_{i} c_{i} \frac{\kappa^{i}}{i !}$ is an even function, while $\sin \mathscr{D} \kappa=: \sum_{i} s_{i} \frac{\kappa^{i}}{i !}$ is odd (Figs. 5 and 6). The flexes of $\mathscr{D}$ are the points $\left(\frac{\sqrt[3]{2}}{2}, \pm \frac{\sqrt[3]{2}}{2}\right)$ and the improper point of Y-axis; the inflectional tangent lines are $x=0$ and $x \pm y=\sqrt[3]{2}$.

In our case, system (7) becomes

$$
\left\{\begin{array}{l}
x^{\prime}=-2 x y, \quad x(0)=1, \\
y^{\prime}=x^{2}+y^{2}, \quad y(0)=0,
\end{array}\right.
$$

from which we can derive that coefficients $c_{i}, s_{i}$ are integers; moreover, the building rule for both matrices $\mathfrak{M}_{\cos \mathscr{D}}$ and $\mathfrak{M}_{\sin _{\mathscr{D}}}$ is

$$
\mathfrak{M}(m, n)=(3 n-2 m-3) \mathfrak{M}(m-1, n-1)+(n+1) \mathfrak{M}(m-1, n+1)
$$

The following table shows some coefficients $c_{i}, s_{i}$ (Table 1), drawn from $\mathfrak{M}_{\cos \mathscr{D}}$, $\mathfrak{M}_{\sin _{\mathscr{D}}}$, computed by means of a common spreadsheet ${ }^{5}$.

\footnotetext{
4 Actually, the curve $\mathscr{D}$ is the image of the Fermat cubic $x^{3}+y^{3}=1$ under the rotation of angle $-\pi / 2$, followed by the homothety of factor $2^{-1 / 6}$.

5 Actually, in order to find $c_{2 i}$ and $s_{2 i-1}$, only $\left(\begin{array}{c}i+1 \\ 2\end{array}\right)-1$ cells of each matrix must be previously filled, so that, for small $i$ those coefficients can be computed "by hand".
} 
Now, let us find an analytic expression of the cosine component $\cos \mathscr{D}$ : differentiating the first equation in (9), and using the identity $x^{3}+3 x y^{2}=1$, we obtain the initial value problem

$$
\left\{\begin{array}{l}
x^{\prime \prime}=\frac{2}{3}\left(1-4 x^{3}\right), \\
x(0)=1, \quad x^{\prime}(0)=0 .
\end{array}\right.
$$

Integrating (10), we obtain the inverse cosine:

$$
\kappa(x)=\frac{\sqrt{3}}{2} \int_{x}^{1} \frac{\mathrm{d} u}{\sqrt{u-u^{4}}} .
$$

Observe that condition $0<x \leq 1$ ensures that cosine is real valued. Using entry 259.50 page 134 of [4], the integral in (11) can be computed, obtaining

$$
\kappa(x)=\sqrt[4]{3} \mathrm{~K}\left(\sin \frac{\pi}{12}\right)-\frac{\sqrt[4]{3}}{2} \mathrm{~F}\left(\operatorname{arcos}\left(\frac{1-(\sqrt{3}+1) x}{1+(\sqrt{3}-1) x}\right), \sin \frac{\pi}{12}\right),
$$

the complete and incomplete elliptic integral of first kind. Inverting, we can solve for $x$ in (12) and obtain

$$
\cos \mathscr{D}(\kappa)=x(\kappa)=\frac{\sqrt{3}+1}{\sqrt{3}+2-\overline{\operatorname{cn}}(\kappa)} \frac{1+\overline{\mathrm{cn}}(\kappa)}{2},
$$

where for short we introduce:

$$
\overline{\operatorname{cn}}(u):=\operatorname{cn}\left(\frac{2}{\sqrt[4]{3}} u, \sin \frac{\pi}{12}\right)
$$

Function $\sin _{\mathscr{D}}$ is obtained using the first equation in (9):

$$
\begin{aligned}
\sin _{\mathscr{D}}(\kappa) & =y(\kappa)=-\frac{1}{2} \frac{x^{\prime}(\kappa)}{x(\kappa)} \\
& =\sqrt[4]{3} \frac{\sqrt{3}+1}{\sqrt{3}+2-\overline{\mathrm{cn}}(\kappa)} \frac{\overline{\operatorname{sn}}(\kappa) \overline{\mathrm{dn}}(\kappa)}{1+\overline{\mathrm{cn}}(\kappa)}
\end{aligned}
$$

where we define:

$$
\overline{\operatorname{sn}}(u):=\operatorname{sn}\left(\frac{2}{\sqrt[4]{3}} u, \sin \frac{\pi}{12}\right), \overline{\operatorname{dn}}(u):=\operatorname{dn}\left(\frac{2}{\sqrt[4]{3}} u, \sin \frac{\pi}{12}\right),
$$

and lastly:

$$
\tan _{\mathscr{D}}(\kappa)=2 \sqrt[4]{3} \frac{\overline{\operatorname{sn}}(\kappa) \overline{\mathrm{dn}}(\kappa)}{(1+\overline{\mathrm{cn}}(\kappa))^{2}}
$$




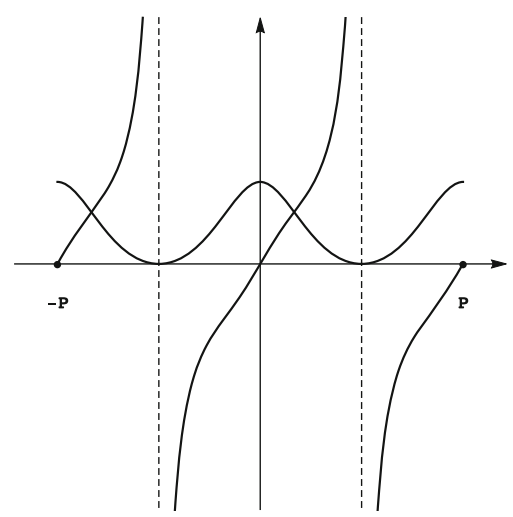

Fig. $6 \cos \mathscr{D}$ and $\sin \mathscr{D}$

Being the curve $\mathscr{D}$ projectively closed, its k-map $\mathbf{m}_{\mathscr{D}}$ is periodic: with some advanced calculus it can be proven that the period is

$$
\Pi_{\mathscr{D}}=2 \pi_{\mathscr{D}}=4 \int_{0}^{1} \sqrt{\frac{1-x^{3}}{3 x}} \mathrm{~d} x=\frac{1}{\sqrt[3]{2}} \mathrm{~B}\left(\frac{1}{3}, \frac{1}{3}\right) .
$$

On the other hand, identities (13) and (14) ensure that the period can also be given in terms of complete elliptic integral of first kind, as

$$
\Pi_{\mathscr{D}}=2 \sqrt[4]{3} \mathrm{~K}\left(\sin \frac{\pi}{12}\right)
$$

in accordance with the fact that $\sin \frac{\pi}{12}$ is a singular modulus (see [20]).

Both the component of $\mathbf{m}_{\mathscr{D}}$ are elliptic functions: then, their second (complex) period can be computed by starting from the classical periodicity relations for Jacobi elliptic functions (see [2] page 39):

$$
\begin{aligned}
& \operatorname{sn}\left(u+2 \mathrm{i} \mathrm{K}^{\prime}\right)=\operatorname{sn} u, \\
& \operatorname{cn}\left(u+2 \mathrm{~K}+2 \mathrm{i} \mathrm{K}^{\prime}\right)=\operatorname{cn} u, \\
& \operatorname{dn}\left(u+4 \mathrm{i} \mathrm{K}^{\prime}\right)=\operatorname{dn} u,
\end{aligned}
$$

where, as usual, $\mathrm{K}^{\prime}(k)=\mathrm{K}\left(k^{\prime}\right)=\mathrm{K}\left(\sqrt{1-k^{2}}\right)$. Thus, the second period is

$$
\Pi_{\mathscr{D}}^{\prime}=\sqrt[4]{3}\left(\mathrm{~K}\left(\sin \frac{\pi}{12}\right)+\mathrm{i} \mathrm{K}\left(\cos \frac{\pi}{12}\right)\right)
$$

finally, by virtue of the singular modulus relation

$$
\mathrm{K}\left(\cos \frac{\pi}{12}\right)=\sqrt{3} \mathrm{~K}\left(\sin \frac{\pi}{12}\right),
$$


Fig. 7 The curve $x^{3}-3 x y^{2}=1$

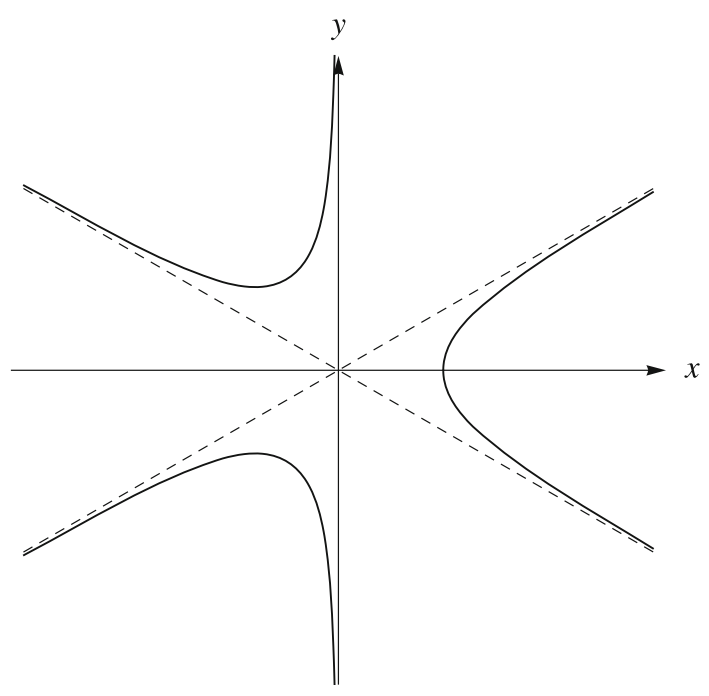

we get

$$
\Pi_{\mathscr{D}}^{\prime}=\frac{1+\mathrm{i} \sqrt{3}}{2} 2 \sqrt[4]{3} \mathrm{~K}\left(\sin \frac{\pi}{12}\right)=\mathrm{e}^{\mathrm{i} \pi / 3} \Pi_{\mathscr{D}}
$$

In closure, the complete lattice $\Lambda_{\mathscr{D}}$ of periods of $\mathbf{m}_{\mathscr{D}}$ is

$$
\Lambda_{\mathscr{D}}=\mathbb{Z} \Pi_{\mathscr{D}} \oplus \mathbb{Z} \mathrm{e}^{\mathrm{i} \pi / 3} \Pi_{\mathscr{D}}
$$

\subsection{The Humbert cubic}

As a second example, let us now study the Keplerian smooth cubic $\mathscr{H}$ of equation

$$
x^{3}-3 x y^{2}-1=0
$$

This cubic was studied by M.G. Humbert, in [12], Sect. 44 Eq. (7), who provided a parametrization in terms of Weierstrass $\wp$ function (Fig. 7).

The curve $\mathscr{H}$, also projectively closed, has symmetry axes $y=0, x= \pm \frac{\sqrt{3}}{3} y$; its inflexion points are improper, with $x=0, y= \pm \frac{\sqrt{3}}{3} x$, as inflexional lines ${ }^{6}$.

In this case, system (7), becomes

$$
\left\{\begin{array}{l}
x^{\prime}=2 x y, \quad x(0)=1, \\
y^{\prime}=x^{2}-y^{2}, \quad y(0)=0 .
\end{array}\right.
$$

\footnotetext{
6 Although $\mathbb{C}$-projectively equivalent, the curves $\mathscr{H}$ and $\mathscr{D}$ are not $\mathbb{R}$-projectively equivalent, as shown by the different arrangement of their inflexional tangents.
} 
By implementing the same process followed in the preceding case, we can find the following analytic expression of the k-map $\mathbf{m}_{\mathscr{H}}$

$$
\begin{aligned}
& \cos \mathscr{H}(\kappa)=\frac{\sqrt{3}-1}{\sqrt{3}-2+\underline{\mathrm{cn}}(\kappa)} \frac{1+\underline{\mathrm{cn}}(\kappa)}{2} \\
& \sin _{\mathscr{H}}(\kappa)=\sqrt[4]{3} \frac{\sqrt{3}-1}{\sqrt{3}-2+\underline{\mathrm{cn}}(\kappa)} \frac{\underline{\operatorname{sn}}(\kappa) \underline{\mathrm{dn}}(\kappa)}{1+\underline{\mathrm{cn}}(\kappa)},
\end{aligned}
$$

where

$$
\begin{aligned}
& \underline{\operatorname{cn}}(u):=\operatorname{cn}\left(\frac{2}{\sqrt[4]{3}} u, \cos \frac{\pi}{12}\right), \\
& \underline{\operatorname{sn}}(u):=\operatorname{sn}\left(\frac{2}{\sqrt[4]{3}} u, \cos \frac{\pi}{12}\right), \\
& \underline{\operatorname{dn}}(u):=\operatorname{dn}\left(\frac{2}{\sqrt[4]{3}} u, \cos \frac{\pi}{12}\right) .
\end{aligned}
$$

Identities (15) and (16) ensure that the map $\mathbf{m}_{\mathscr{H}}$ is periodic, with period

$$
\Pi_{\mathscr{H}}=2 \sqrt[4]{3} \mathrm{~K}\left(\cos \frac{\pi}{12}\right)=\sqrt{3} \Pi_{\mathscr{D}}
$$

By considering that $\mathscr{H}$ is projectively closed, we can claim that the period of its kmap must equal twelve times the area of the region bounded by the curve and the lines $x=0$ and $y=\frac{1}{\sqrt{3}} x$; more precisely, accordingly to the fact that $\cos \frac{\pi}{12}$ is a singular modulus,

$$
\Pi_{\mathscr{H}}=12\left(\frac{1}{2 \sqrt{3}}+\int_{1}^{\infty}\left(\frac{1}{\sqrt{3}} x-\sqrt{\frac{x^{3}-1}{3 x}}\right) \mathrm{d} x\right)=\frac{\sqrt{3}}{2} \mathrm{~B}\left(\frac{1}{3}, \frac{1}{6}\right) .
$$

Also in this case, the components of $\mathbf{m}_{\mathscr{H}}$ are both elliptic functions, with complex period

$$
\begin{aligned}
\Pi_{\mathscr{H}}^{\prime} & =\frac{\sqrt{3}+\mathrm{i}}{2} \frac{2}{\sqrt[4]{3}} \mathrm{~K}\left(\cos \frac{\pi}{12}\right) \\
& =\mathrm{e}^{\mathrm{i} \pi / 6} \Pi_{\mathscr{D}} .
\end{aligned}
$$

Setting now

$$
\left[\begin{array}{l}
\Pi_{\mathscr{H}}^{\prime \prime} \\
\Pi_{\mathscr{H}}^{\prime \prime \prime}
\end{array}\right]:=\left[\begin{array}{ll}
-1 & 2 \\
-1 & 1
\end{array}\right]\left[\begin{array}{l}
\Pi_{\mathscr{H}} \\
\Pi_{\mathscr{H}}^{\prime}
\end{array}\right]
$$

we realize that $\Pi_{\mathscr{H}}^{\prime \prime}$ and $\Pi_{\mathscr{H}}^{\prime \prime \prime}$ generate the complete lattice $\Lambda \mathscr{H}$ of periods of $\mathbf{m} \mathscr{H}$ :

$$
\Lambda_{\mathscr{H}}=\mathbb{Z} \Pi_{\mathscr{H}}^{\prime \prime} \oplus \mathbb{Z} \Pi_{\mathscr{H}}^{\prime \prime \prime} .
$$


But, in addition, the identity

$$
\left[\begin{array}{l}
\Pi_{\mathscr{H}}^{\prime \prime} \\
\Pi_{\mathscr{H}}^{\prime \prime \prime}
\end{array}\right]=\mathrm{i}\left[\begin{array}{l}
\Pi_{\mathscr{D}} \\
\Pi_{\mathscr{D}}^{\prime}
\end{array}\right]
$$

proves that the lattice $\Lambda_{\mathscr{H}}$ is congruent to $\Lambda_{\mathscr{D}}$, via the rotation by $\pi / 2$.

\section{A final remark}

All arguments outlined in these pages can apply for a wider class of parametric curves, by leaving out axiom (AnK 0). Actually, that axiom simply succeeds to exhibit the " trigonometric" behaviour of a k-map, by displaying $\mathbf{m}(0)=\mathbf{i}$ : its removal must be remedied by giving the role of $\mathbf{m}(0)$ to a point picked at will in the assigned curve.

Funding Open Access funding provided by Alma Mater Studiorum - Università di Bologna. Work supported by RFO 2015-2016 (Panel 13) Italian grant funding.

\section{Compliance with ethical standards}

Conflicts of Interest Statement The author declares that he has no known competing financial interests or personal relationships that could have appeared to influence the work reported in this paper.

Open Access This article is licensed under a Creative Commons Attribution 4.0 International License, which permits use, sharing, adaptation, distribution and reproduction in any medium or format, as long as you give appropriate credit to the original author(s) and the source, provide a link to the Creative Commons licence, and indicate if changes were made. The images or other third party material in this article are included in the article's Creative Commons licence, unless indicated otherwise in a credit line to the material. If material is not included in the article's Creative Commons licence and your intended use is not permitted by statutory regulation or exceeds the permitted use, you will need to obtain permission directly from the copyright holder. To view a copy of this licence, visit http://creativecommons.org/licenses/by/4.0/.

\section{References}

1. Adams, O.: Elliptic functions applied to conformal world maps. Washington Government printing office, Washington (1925)

2. Bowman, F.: Introduction to Elliptic Functions, with applications. English University Press, London (1953)

3. Burgoyne, F.: Generalized trigonometric functions. Math. Comput. 18(86), 314-316 (1964). https:// doi.org/10.2307/2003310

4. Byrd, P., Friedman, M.: Handbook of Elliptic Integrals for Engineers and Scientists. Springer, New York (1971)

5. Cayley, A.: On the elliptic function solution of the equation $x^{3}+y^{3}-1=0$. Proc. Cambridge Philos Soc 4, 106-109 (1883)

6. Dixon, A.: The elementary properties of the elliptic functions. MacMillian and Co., New York (1894)

7. Edmunds, D., Gurka, P., Lang, J.: Properties of generalized trigonometric functions. J. Approx. Theory 164(1), 47-56 (2012). https://doi.org/10.1016/j.jat.2011.09.004

8. Edmunds, D., Gurka, P., Lang, J.: Basis properties of generalized trigonometric functions. J. Math. Anal. Appl. 420(2), 1680-1692 (2014). https://doi.org/10.1016/j.jmaa.2014.06.015

9. Elbert, A.: Colloq. Math. Soc. János Bolyai, vol. 30, chap. A half-linear second order differential equation, pp. 158-180. North-Holland, Amsterdam-New York (1979) 
10. Girg, P., Kotrla, L.: p-Trigonometric and $p$-Hyperbolic Functions in Complex Domain. Abstract Appl. Anal. (2016). https://doi.org/10.1155/2016/3249439

11. Grammel, R.: Eine Verallgemeinerung der Kreis-und Hyperbelfunktionen. Arch. Appl. Mech. 16(3), 188-200 (1948)

12. Humbert, M.: Sur le théorème d'Abel. Troisieme partie: Des courbes de direction. J. Math. Pures Appl. pp. 371-404 (1887)

13. Lang, J., Edmunds, D.: Eigenvalues, Embeddings and Generalised Trigonometric Eigenvalues, Embeddings and Generalised Trigonometric Functions. Lecture Notes in Math, vol. 2016. Springer, Berlin (2011)

14. Lindqvist, P.: Some remarkable sine and cosine functions. Ricerche di Matematica 44(2), 269-290 (1995)

15. Lindqvist, P., Peetre, J.: Erik Lundberg och de hypergoniometriska funktionerna. Normat 45(1), 1-24 (1997)

16. Lindqvist, P., Peetre, J.: $p$-arclength of the $q$-circle. Math. Student 72, 139-145 (2003)

17. Lindqvist, P., Peetre, J.: Comments on Erik Lundberg's 1879 thesis, especially on the work of Göran Dillner and his influence on Lundberg. Istituto Lombardo Acad. Sci. Lett., Classe Sci. Mat. Nat, Mem (2004). https://doi.org/10.2307/2695794

18. Poodiack, R.D.: Squigonometry, Hyperellipses, and Supereggs. Math. Mag. 89(2), 92-102 (2016). https://doi.org/10.4169/math.mag.89.2.92

19. Robinson, P.L.: The Dixonian ellpitic function. arxiv preprint (2019)

20. Selberg, A., Chowla, S.: On Epstein's zeta-function. J. Reine Angew. Math 227(86), 110 (1967)

21. Shelupsky, D.: A generalization of the trigonometric functions. Am. Math. Monthly 66(10), 879-884 (1959). https://doi.org/10.1080/00029890.1959.11989425

22. Takeuchi, S.: Multiple-angle formulas of generalized trigonometric functions with two parameters. J. Math. Anal. Appl. 444(2), 1000-1014 (2016). https://doi.org/10.1016/j.jmaa.2016.06.074

23. Wood, W.: Squigonometry. Math. Mag. 84(4), 257-265 (2011). https://doi.org/10.4169/math.mag.84. 4.257

24. van Fossen Conrad, E., Flajolet, P.: The Fermat cubic, elliptic functions, continued fractions, and a combinatorial excursion. Séminaire Lotharingien de Combinatoire 54(B54g), 1-44 (2006)

Publisher's Note Springer Nature remains neutral with regard to jurisdictional claims in published maps and institutional affiliations. 